\title{
INCREASING THE LUMINOSITY WITH THE BEAM-BEAM INTERACTION LUMINOSITY MONITOR*
}

\author{
D. Sagan \\ Wilson Laboratory, Cornell University, Ithaca NY, 14853, USA
}

\begin{abstract}
At the Cornell Electron/positron Storage Ring CESR, a fast luminosity monitor has been implemented[1]. The monitor works by measuring the amplitude of oscillation of a given bunch while the corresponding opposing bunch is shaken. A disadvantage with the luminosity monitor is that the monitor signal, besides being a measure of the luminosity, is also dependent upon other factors such as the vertical tune and the vertical beta function at the IP. To overcome this deficiency, a software program has been developed to give a normalized signal that only depends upon the specific luminosity. In HEP conditions, this normalized signal is used as input to an "optimizer" program that automatically varies machine parameters to keep the luminosity at a maximum.
\end{abstract}

\section{INTRODUCTION}

In a colliding beam storage ring it is essential to be able to monitor the luminosity so as to be able to adjust machine elements (magnets, separators, etc.) to maximize the luminosity. Recently, a fast luminosity monitor has been developed at the Cornell Electron/positron Storage Ring CESR[1]. The monitor is shown schematically in Fig. 1. Selected bunches of one beam are shaken vertically at a given frequency. These "shaker" bunches shake the corresponding "detected" bunches at the interaction point (IP), and the amplitude of oscillation of the detected bunches are then monitored, via a BPM detector, by a lock-in amplifier. In standard practice at CESR, the amplitude of the shaker bunch is only a few percent of the vertical beam size. Thus, the operation of the monitor has no significant affect on operations. While the amplitude of oscillation of the detected bunches at the BPM is of order $1 \mu \mathrm{m}$, and the monitor signal level is of order $100 \mu \mathrm{V}$, the inherent noise rejection of the lock-in gives an excellent signal-to-noise ratio. Typically, for a 1 second time constant, the signalto-noise ratio is 100 to 1 or better. A problem with the monitor, though, is that the monitor signal is not simply proportional to the luminosity, but is dependent upon other factors as well. For head-on collisions, the monitor signal $S$ is proportional to[1]

$$
S \propto \mathcal{L} \beta_{y}(\mathrm{ip}) \sqrt{\beta_{y}(\mathrm{sh}) \beta_{y}(\operatorname{det})} F_{s h} F_{\text {det }} / I,
$$

\footnotetext{
* Work supported by the National Science Foundation
}

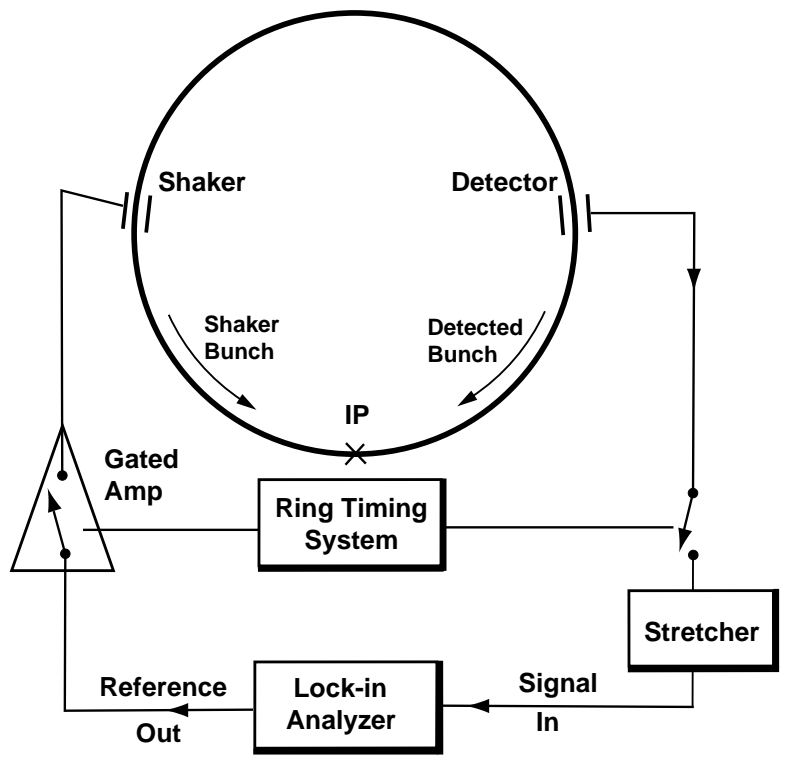

Figure 1: Schematic diagram of the BBI luminosity monitor configuration.

where $\mathcal{L}$ is the luminosity, $I$ is the bunch current, and $F_{\text {sh }}$ and $F_{d e t}$ are defined by

$$
F_{s h}\left(Q_{y}, Q_{s}, \phi_{y}(\mathrm{sh})\right) \equiv \frac{A_{s}(\mathrm{ip})}{A_{s}^{\prime}(\mathrm{sh}) \sqrt{\beta_{y}(\mathrm{sh}) \beta_{y}(\mathrm{ip})}}
$$

and

$$
F_{\text {det }}\left(Q_{y}, Q_{s}, \phi_{y}(\operatorname{det})\right) \equiv \frac{A_{d}(\operatorname{det})}{A_{d}^{\prime}(\mathrm{ip}) \sqrt{\beta_{y}(\mathrm{ip}) \beta_{y}(\operatorname{det})}}
$$

Here $A_{s}^{\prime}(\mathrm{sh})$ is the amplitude of the kick given the skaker bunch at the shaker, $A_{s}(\mathrm{ip})$ is the amplitude of the shaker bunch oscillations at the IP, $A_{d}^{\prime}(\mathrm{ip})$ is the amplitude of the kick given the detected bunch at the IP, and $A_{d}(\mathrm{det})$ is the amplitude of the detected bunch oscillations at the detector.

$F_{s h}\left(Q_{y}, Q_{s}, \phi_{y}(\mathrm{sh})\right)$ depends upon the vertical tune $Q_{y}$, the frequency of shaking $Q_{s}$, and the phase advance between the shaker and the IP $\phi_{y}(\mathrm{sh})$ [2]. Similarly, $F_{\text {det }}\left(Q_{y}, Q_{s}, \phi_{y}(\mathrm{det})\right)$ is dependent upon $Q_{y}, Q_{s}$ and $\phi_{y}(\mathrm{det})$ - the phase advance between the detector and the IP. 


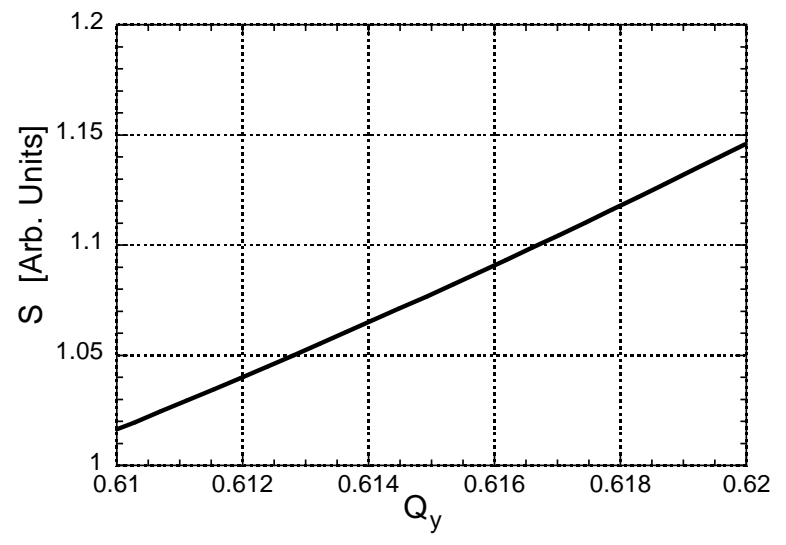

Figure 2: Variation of $S$ as a function of vertical tune with the luminosity and horizontal tune held constant.

\begin{tabular}{|l|l||l|l|}
\hline Parameter & Value & Parameter & Value \\
\hline$Q_{s}$ & $0.26(100 \mathrm{kHz})$ & $Q_{y}$ & 0.61 \\
\hline$A_{s}^{\prime}(\mathrm{sh})$ & $0.5 \mu \mathrm{rad}$ & $\xi_{y}$ & 0.03 \\
\hline$\beta_{y}(\mathrm{ip})$ & $0.019 \mathrm{~m}$ & $\sigma_{y}(\mathrm{ip})$ & $7 \mu \mathrm{m}$ \\
\hline$\beta_{y}(\mathrm{sh})$ & $21.5 \mathrm{~m}$ & $\beta_{y}($ det $)$ & $32.3 \mathrm{~m}$ \\
\hline$\phi_{y}(\mathrm{sh})$ & $2 \pi \cdot 0.86$ & $\phi_{y}($ det $)$ & $2 \pi \cdot 0.27$ \\
\hline$F_{s h}$ & 0.79 & $F_{\text {det }}$ & 0.82 \\
\hline
\end{tabular}

Table 1: CESR BBI luminosity monitor parameters.

\section{NORMALIZATION PROGRAM}

An example of how the monitor signal is dependent upon things other than the luminosity is shown in Figure 2. Figure 2 shows $S$, as calculated from Eq. (1), as a function of vertical tune while holding the luminosity and the horizontal tune constant. Parameters used in the computation are typical of CESR high energy physics (HEP) conditions and are given in Table 1 . The change in $Q_{y}$ is simulated by varying the strengths of the quadrupoles in the arcs to give minimal change in the beta functions. Over the tune range of the graph, which is typical of the range over which the tune can be varied during an HEP run, $S$ varies of order $10 \%$. This variation can be larger than the actual variation of the luminosity. The trend of $S$ in the figure can be simply understood as a resonance phenomena by noting that, as the tune is increased, the aliased tune, $1-Q_{y}$, is moving nearer the shaking frequency at $Q_{s}=0.26$.

In order to overcome the dependency of the monitor signal on the vertical betas and phases, a software program has been developed that normalizes the signal to give a quantity that, at least in theory, is directly a measure of the specific luminosity. The normalization program works by using Eq. (1) to compute the "predicted" signal $S_{p}$ assuming constant $\mathcal{L} / I$. With this, the normalized signal $S_{n}$ is given by

$$
S_{n}=\frac{S}{S_{p} I}
$$

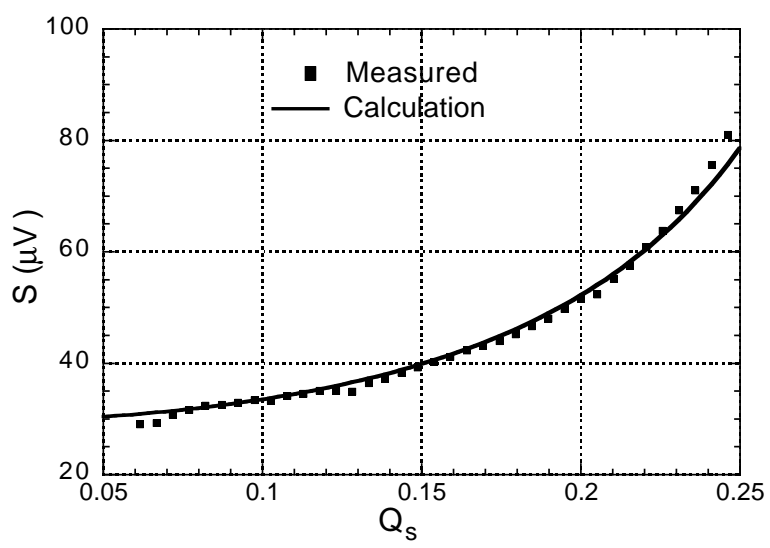

Figure 3: Calculated and measured monitor signal as a function of shaker frequency. With the calculated $S$ there is one adjustable constant that gives the overall gain of the system. This constant was chosen to best match the measured results.

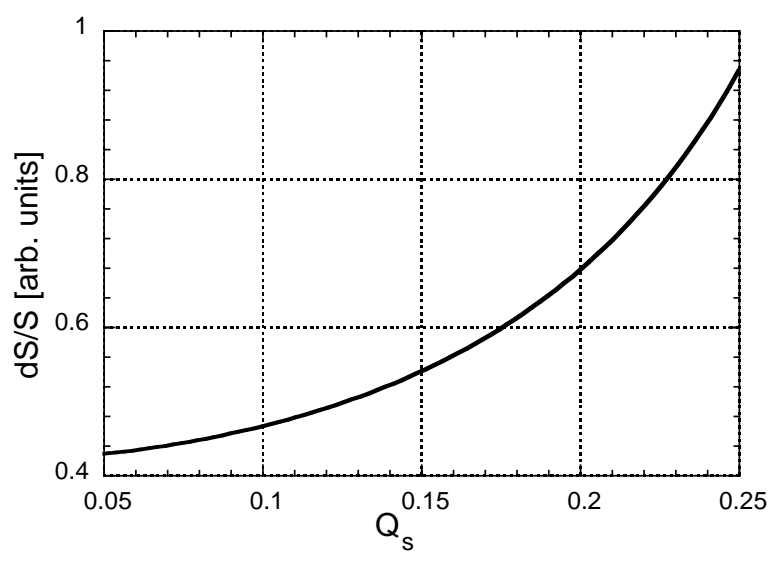

Figure 4: Relative Sensitivity of the monitor signal to changes in vertical tune as a function of shaker frequency. $d S$ is the change in $S$, for a fixed change in $Q_{y}$, as computed from Eq. (1).

At the start of a HEP run, the values for the quadrupole strengths, quadrupole rotation angles, and sextupole strengths[3] are read in from the CESR data base. It is assumed that these initial settings correspond to the settings for the theoretical design lattice. That is, the initial Twiss parameters are assumed equal to the Twiss parameters of the design lattice. $S_{p}$ is computed assuming a constant value for $\mathcal{L} / I$ with head-on collisions.

The program recomputes $S_{n}$ approximately 8 times a second and puts this number in the CESR data base for general use. To save on computation time, the database is monitored, and only if any of the quadrupole or sextupole settings have changed are the Twiss parameters and $S_{p}$ recomputed. This recomputation is based upon the change in 
the settings and the known calibration constants.

The working of the normalization program rests on the assumption that Eq. (1) accurately reflects reality. A good indication that this is so is shown by the good agreement between measured and calculated monitor signal levels as a function of shaking frequency $Q_{s}$. This is shown in Figure 3. The greatest weakness in the above procedure is the assumption that the initial conditions correspond to the design lattice. This assumption is necessary since hysteresis in the magnets precludes an accurate enough absolute calibration. In practice, the assumption is generally close enough so that the normalized signal generally follows the luminosity. One way to desensitize the signal dependence on tune would be to lower $Q_{s}$ as shown in Figure 4. Figure 4 shows, as a function of $Q_{s}$, the quantity $d S / S-$ the change in $S$, for a fixed change in $Q_{y}$, normalized by $S$. Lowering $Q_{s}$, however, has the drawback of lowering $S$ (cf. Figure 3) since, currently at CESR, the shaker driver amplifier is near maximum output and so $A_{s}^{\prime}(\mathrm{sh})$ cannot be increased. Moreover, lowering $Q_{s}$ does nothing to lessen the dependence on the betas.

\section{OPTIMIZATION PROGRAM}

At CESR, the normalized luminosity signal is used with an "optimizer" program to provide automatic adjustment of machine parameters to maximize the luminosity. The optimizer program is a general purpose program in that it can be used to maximize (or minimize) any quantity that is stored in the CESR data base using any control variables in the data base. Several optimizing strategies are available for use. However, for maximizing the luminosity, the favorite strategy used involves varying a variable back and forth over some range while recording the normalized signal. To reduce the noise, the signal vs. variable curve is smoothed using a sliding window and the maximum of the curve is found. The variable is then set to the value corresponding to the maximum and the cycle is started over with the next variable on the list of variables to use.

The use of the optimizer has lead to some puzzling behavior. It has been found that, at the start of a HEP run, the optimum conditions have a small but significant coupling component in the arcs. Why this should be so is so far unexplained, but one clue is that, as the run progresses, and the beam current decays, the optimizer steadily decreases the coupling by reducing the strength of skew quadrupoles under its control. This strongly suggests that the explanation involves the beam-beam interaction.

\section{CONCLUSION}

Without the normalization program, the beam-beam interaction luminosity monitor was useful for making sure that the beams collided head-on and for adjusting skew quadrupoles for minimum beam size — both of which did not depend upon variation of the Twiss parameters. With the normalization program, the range of usefulness has been extended to the ability to adjust quadrupole or sextupole settings. With this, the luminosity monitor, along with the optimizer program, is in standard use at CESR.

\section{ACKNOWLEDGEMENTS}

My thanks to Stu Henderson and John Sikora for helping with the beam-beam interaction luminosity monitor and to the rest of the CESR operations group for their support. Especial thanks to Flora Sagan for her editorial support.

\section{REFERENCES}

[1] D. Sagan, J. Sikora, and S. Henderson, "A Luminosity Monitor using the Coherent Beam-Beam Interaction," Proc. 1997 Part. Acc. Conf., pg. 1765 (1997).

[2] there is also a dependence upon the damping decrement but this dependence is small away from resonance and can be ignored.

[3] Since a pretzeled orbit is used to separate the beams (which share a common beam pipe), the sextupoles affect the Twiss parameters and thus need to be monitored. 